\title{
Observing the continental-scale carbon balance: assessment of sampling complementarity and redundancy in a terrestrial assimilation system by means of quantitative network design
}

\author{
T. Kaminski ${ }^{1}$, P. J. Rayner ${ }^{2}$, M. Voßbeck ${ }^{1}$, M. Scholze ${ }^{3}$, and E. Koffi ${ }^{4}$ \\ ${ }^{1}$ FastOpt, Lerchenstraße 28a, 22767 Hamburg, Germany \\ ${ }^{2}$ School of Earth Sciences, University of Melbourne, Melbourne, Australia \\ ${ }^{3}$ Dept. of Earth Sciences, University of Bristol, Bristol, UK \\ ${ }^{4}$ LSCE/IPSL, Gif-sur-Yvette Cedex, France \\ Correspondence to: T. Kaminski (thomas.kaminski@fastopt.com)
}

Received: 29 February 2012 - Published in Atmos. Chem. Phys. Discuss.: 12 March 2012

Revised: 31 July 2012 - Accepted: 17 August 2012 - Published: 31 August 2012

\begin{abstract}
This paper investigates the relationship between the heterogeneity of the terrestrial carbon cycle and the optimal design of observing networks to constrain it. We combine the methods of quantitative network design and carboncycle data assimilation to a hierarchy of increasingly heterogeneous descriptions of the European terrestrial biosphere as indicated by increasing diversity of plant functional types. We employ three types of observations, flask measurements of $\mathrm{CO}_{2}$ concentrations, continuous measurements of $\mathrm{CO}_{2}$ and pointwise measurements of $\mathrm{CO}_{2}$ flux. We show that flux measurements are extremely efficient for relatively homogeneous situations but not robust against increasing or unknown complexity. Here a hybrid approach is necessary, and we recommend its use in the development of integrated carbon observing systems.
\end{abstract}

\section{Introduction}

$\mathrm{CO}_{2}$ and methane are the most important anthropogenic greenhouse gases. Their increasing concentration is the major reason for global warming (Solomon et al., 2007). It is thus of paramount interest to quantify and ultimately predict the exchanges of these gases between the terrestrial biosphere and the atmosphere. At a number of points on the globe, carbon and water fluxes are sampled directly (see, e.g. http://www.fluxnet.ornl.gov). The interpolation of these measurements to the globe (upscaling) requires external in- formation about the uncertain spatio-temporal flux structure. The same type of information is required by atmospheric transport inversions (see e.g. Rayner et al., 1999; Gurney et al., 2002; Enting, 2002) which infer surface fluxes from atmospheric concentration measurements. The most sophisticated tools for quantifying the structure and variability of carbon fluxes are process models of the terrestrial carbon cycle like those used for the assessments of the IPCC (Solomon et al., 2007). Underlying these models is the assumption of fundamental equations that govern the processes controlling the terrestrial carbon fluxes. Uncertainty in the simulation of these fluxes arises from four sources: first, there is uncertainty in the forcing data (such as precipitation or temperature) driving the terrestrial processes. Second, there is uncertainty regarding the formulation of individual processes and their numerical implementation (structural uncertainty). Third, there are uncertain constants (process parameters) in the formulation of these processes (parametric uncertainty). Forth, there is uncertainty about the state of the terrestrial biosphere at the beginning of the simulation.

Observational information helps to reduce these uncertainties. Currently there are several initiatives to extend the observational network of the carbon cycle. Europe's Integrated Carbon Observing System (ICOS, see http://www. icos-infrastructure.eu/), for example, aims at setting up an integrated sampling network for land, ocean, and atmosphere. Ideally, all data streams are interpreted simultaneously with the process information provided by the model to yield a 
consistent picture of the carbon cycle that balances all the observational constraints, thereby taking the respective uncertainty ranges into account. Data assimilation systems around prognostic models of the carbon cycle are the ideal tools for this integration allowing us to assimilate a wide range of observations. They can, for example, be applied to systematically reduce parametric uncertainty (e.g. Kaminski et al., 2002) or to expose structural errors (Rayner, 2010). In a first step, they use the observations to constrain the uncertain process parameters (calibration), and in a second step they use the calibrated model for analysis and prediction. Ideally, both steps include the propagation of uncertainties. This allows us to derive uncertainty ranges on simulated target quantities that are consistent with the uncertainties in the observations and the model. Examples of such target quantities are fluxes of carbon on regional, continental, or global scale, integrated over part of the assimilation period (diagnostic target quantity) or some period before or after (prognostic target quantity). With regard to a specified target quantity, such an assimilation system can assess the performance of a given observational network. This performance is typically quantified by the uncertainty range.

Quantitative Network Design (QND) aims at constructing an observational network with optimal performance. The approach is based on Hardt and Scherbaum (1994) who optimised the station locations for a seismographic network. It was first applied to observational networks of the global carbon cycle by Rayner et al. (1996), who optimised the locations of atmospheric $\mathrm{CO}_{2}$ and $\delta^{13} \mathrm{C}$ measurements. $\mathrm{A}$ pioneering study for sensor design has been performed by Rayner and O'Brien (2001) who established the required precision for observations of the column-integrated atmospheric $\mathrm{CO}_{2}$ concentration from space.

The latter two studies investigated purely atmospheric networks. To assist the design of an integrated carbon observing system, we need the capability of evaluating the complementarity of various observational data streams including those of the terrestrial biosphere. As outlined by Kaminski and Rayner (2008) assimilation systems are the ideal tool for this task. The Carbon Cycle Data Assimilation System (CCDAS, see http://ccdas.org) can assimilate several observational data streams and infers uncertainty ranges on diagnosed (Rayner et al., 2005) or prognosed carbon (Scholze et al., 2007; Rayner et al., 2011) and water (Kaminski et al., 2012) fluxes. The first QND applications investigated the utility of space borne observations of atmospheric $\mathrm{CO}_{2}$ (Kaminski et al., 2010) or vegetation activity (Kaminski et al., 2012) in constraining various surface fluxes. Another study explores the atmospheric in situ network and its ability to constrain the productivity of the terrestrial biosphere (Koffi et al., 2012).

Kaminski and Rayner (2008) noted two general aspects of QND studies. The first is the dependence on the target quantity; clearly different networks are optimal for constraining different things (Rayner et al., 1996). The second is the dependence on prior knowledge brought to the problem. For traditional inversions of fluxes this information takes the form of the covariance of prior uncertainty. For CCDAS it is determined by the process resolution of the underlying dynamical model (how many processes are modelled) and the spatial detail at which these processes are allowed to vary independently. The level of heterogeneity of the biosphere is a fundamental question which goes beyond CCDAS; it determines how much any understanding of processes gained locally can be more widely applied. However it is clear that observing networks presupposing a given heterogeneity are at some risk. Current earth system models map this spatial heterogeneity by dividing the global vegetation into a small number of plant functional types (PFTs). Groenendijk et al. (2011) demonstrate through calibration of a terrestrial model against direct flux measurements the limit of this approximation and the difficulty in deriving a realistic PFT classification.

This paper uses the network designer, a CCDAS-based interactive QND tool, to investigate the performance of several networks composed of direct flux observations and flask or continuous samples of the atmospheric carbon dioxide concentration. In particular we investigate the robustness of network performance to various choices of target quantities and levels of heterogeneity. The outline of the paper is as follows. Section 2 describes our QND methodology and Sect. 3 the networks we consider. Then Sect. 4 will present and discuss the evaluations. Finally, in Sect. 5 we summarise our conclusions.

\section{Methods}

CCDAS is built around the Biosphere Energy Transfer HYdrology scheme (BETHY, Knorr, 2000; Knorr and Heimann, 2001), a global model of the terrestrial vegetation. The version used here is described in Rayner et al. (2005). This section gives brief descriptions of BETHY, the observational data types, CCDAS and of the QND approach.

\subsection{BETHY}

Following Wilson and Henderson-Sellers (1985) BETHY decomposes the global terrestrial vegetation into 13 PFTs as listed in Table 1. Each grid cell can be covered by up to three PFTs. Figure 1 shows the distribution of the dominant PFT. As in Scholze et al. (2007) we integrate the model over $21 \mathrm{yr}$ from 1979 to 1999 on a global 2 by 2 degree grid and use observed meteorological driving data (Nijssen et al., 2001).

The process formulations within BETHY are controlled by a set of process parameters (see Table 2). For this study we use the model version of Scholze et al. (2007) with the extension of simulating hourly Net Ecosystem Productivity (NEP). This is done by dividing the daily calculated heterotrophic respiration flux into 24 equal-sized hourly fluxes and subtracting these fluxes from the hourly simulated Net 


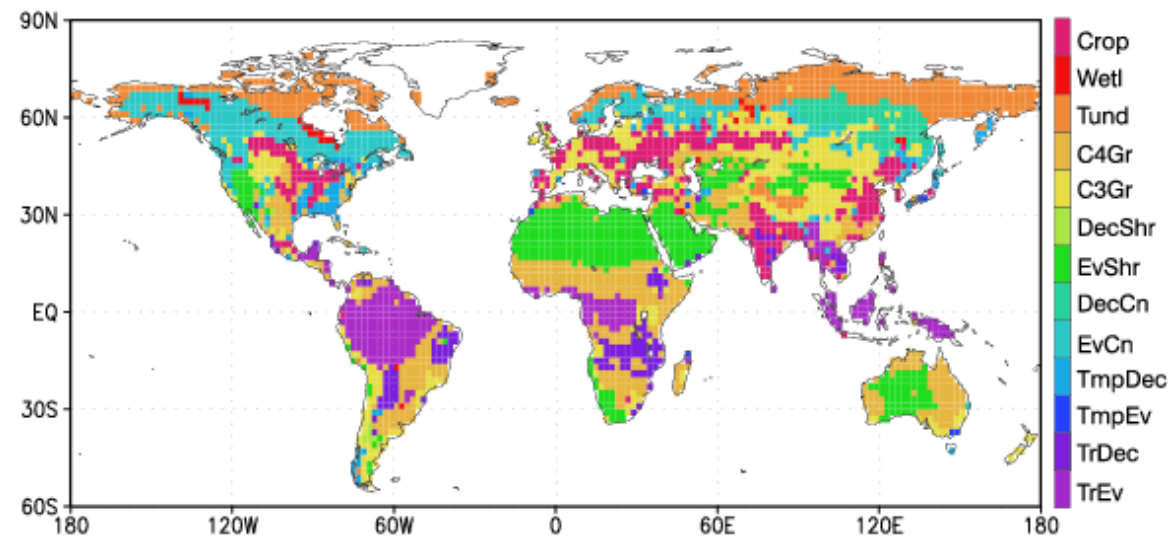

Fig. 1. Distribution of the dominant CCDAS Plant Functional Type (PFT) per grid cell, PFT labels are given in Table 1, taken from Rayner et al. (2005).

Table 1. Plant Functional Types (PFTs) defined in CCDAS and their abbreviations, taken from Rayner et al. (2005).

\begin{tabular}{lll}
\hline PFT No. & PFT Name & Abbreviation \\
\hline 0 & Not vegetated & \\
1 & Trop. broadleaved evergreen tree & TrEv \\
2 & Trop. broadleaved deciduous tree & TrDec \\
3 & Temp. broadleaved evergreen tree & TmpEv \\
4 & Temp. broadleaved deciduous tree & TmpDec \\
5 & Evergreen coniferous tree & EvCn \\
6 & Deciduous coniferous tree & DecCn \\
7 & Evergreen shrub & EvShr \\
8 & Deciduous shrub & DecShr \\
9 & C3 grass & C3Gr \\
10 & C4 grass & C4Gr \\
11 & Tundra vegetation & Tund \\
12 & Swamp vegetation & Wetl \\
13 & Crops & Crop \\
\hline
\end{tabular}

Primary Productivity (NPP). BETHY simulates 13 PFTs including 21 different parameters. Three of these parameters are PFT-specific and 18 are applied globally, i.e. they refer to all PFTs. We thus have $18+3 \times 13=57$ parameters. The role of the individual parameters is described elsewhere (Rayner et al., 2005; Scholze et al., 2007). In our context of network design it is important to know to which parameters our respective target quantities are sensitive. We will use regional integrals of the NPP and the NEP as target quantities. The latter is net $\mathrm{CO}_{2}$ flux between the atmosphere and the biosphere and defined as the difference of NPP and heterotrophic soil respiration. Except for one atmospheric parameter $c_{0}$, all parameters impact NEP. NPP is sensitive to all parameters, except $c_{0}$ and the soil and carbon balance parameters.

\subsection{Observational data types}

In this study we use three types of observational data: direct (NEP) flux measurements, flask and continuous samples of the atmospheric $\mathrm{CO}_{2}$ concentration. Within the model, a flux measurement is represented by a time series of hourly NEP samples of the grid cell the site is located in. The atmospheric data types require, as a so-called observation operator, an atmospheric transport model to transform the global NEP field into atmospheric concentrations. Flask samples are represented by a time series of monthly mean concentrations at the sampling location as simulated by the atmospheric transport model TM2 (Heimann, 1995), which is run at 8 by 10 degree horizontal resolution and with nine vertical levels. As in Carouge et al. (2010a,b) continuous samples are represented by a time series of daily mean concentrations at the sampling location as simulated by the atmospheric transport model LMDZ (Hauglustaine et al., 2004), which is run at 3.75 by 2.5 degree resolution over most of the globe but a zoomed 0.5 degree resolution over Europe. For each data type the observational time series covers the $20 \mathrm{yr}$ period from 1980 to 1999 . By representing flask samples in the model as monthly means, much of the synoptic signal is averaged out. Likewise by representing continuous measurements by daily means the diurnal signal is averaged out. This averaging reduces the information content of the observations but is also less demanding of the models' performance, i.e. a conservative choice. A model setup with enhanced temporal representation of the flask or continuous data types would probably provide stronger constraints on the target quantities.

\subsection{CCDAS}

CCDAS uses a gradient method to adjust BETHY's process parameters in order to minimise a cost function. This cost function quantifies the fit to all observations plus the 
deviation from prior knowledge on the process parameters:

$$
\begin{aligned}
J(\tilde{\mathbf{x}}) & =\frac{1}{2}\left[(M(\tilde{\mathbf{x}})-\mathbf{d})^{T} \mathbf{C}(d)^{-1}(M(\tilde{\mathbf{x}})-\mathbf{d})\right. \\
& \left.+\left(\tilde{\mathbf{x}}-\mathbf{x}_{\mathbf{0}}\right)^{T} \mathbf{C}\left(x_{0}\right)^{-1}\left(\tilde{\mathbf{x}}-\mathbf{x}_{\mathbf{0}}\right)\right]
\end{aligned}
$$

where $M$ denotes the model considered as a mapping from parameters to observations, $d$ the observations with data uncertainty $\mathbf{C}(d), x_{0}$ the prior parameter values with uncertainty $\mathbf{C}\left(x_{0}\right)$, and the superscript $T$ the transpose.

The second derivative (Hessian) of the cost function at the optimum $x$ is used to approximate the inverse of the covariance matrix $\mathbf{C}(x)$ that quantifies the uncertainty ranges on the parameters that are consistent with uncertainties in the observations and the model. In a second step, the linearisation $N^{\prime}$ (Jacobian) of the model $N$ used as a mapping from parameters to target quantities is used to propagate the parameter uncertainties forward to the uncertainty in a target quantity $\sigma(y)$ :

$\sigma(y)^{2}=\boldsymbol{N}^{\prime} \mathbf{C}(x) \boldsymbol{N}^{\prime T}+\sigma\left(y_{\mathrm{mod}}\right)^{2}$.

$\sigma\left(y_{\bmod }\right)$ quantifies all uncertainty in the simulation of the target quantity except the uncertainty in $x$ (which we resolve explicitly). If the terrestrial model was perfect, $\sigma\left(y_{\text {mod }}\right)$ would be zero. In contrast, if the parameters were perfectly known, the first term on the right hand side would be zero. Likewise the data uncertainty $\mathbf{C}(d)$ is the sum of the observational uncertainty and all uncertainty in the simulation of the observations except the uncertainty in the parameter vector.

All derivative information is provided with the same numerical accuracy as the original model in an efficient form via automatic differentiation of the model code by the automatic differentiation tool TAF (Giering and Kaminski, 1998).

\subsection{QND}

In network design mode, CCDAS is restricted to the uncertainty propagation for candidate networks. It builds on the optimal parameter set estimated from data of the available network for the evaluation of the required first and second derivatives. In our case the optimal parameter vector is taken from the study of Scholze et al. (2007). For the evaluation of potential networks, the Hessian is evaluated for $d=M(x)$. In this case the posterior target uncertainty solely depends on the prior and data uncertainties and linearised model responses at observational locations and for target quantities. The approach does not require real observations, and can thus evaluate hypothetical candidate networks (see Kaminski and Rayner, 2008; Kaminski et al., 2010). Candidate networks are defined by a set of observations characterised by observational data type, location, and data uncertainty. In practise for pre-defined target quantities and observational types and locations, model sensitivities can be pre-computed and stored. A network composed of these pre-defined observations, can then be evaluated in terms of the pre-defined target quantities without further model evaluations. Only matrix algebra is required to combine the pre-computed sensitivities with the data uncertainties.

This is the approach implemented in the network designer (see http://imecc.ccdas.org), an interactive software tool that evaluates networks composed of flask and continuous samples of atmospheric $\mathrm{CO}_{2}$ and direct flux measurements. Available target quantities are NPP and NEP over three regions: Europe, Brazil, and Russia (see Fig. 2). They are provided in the form of annual mean values averaged over the $20 \mathrm{yr}$ assimilation period. Model sensitivities have been pre-computed for a list of atmospheric sampling sites (see Fig. 3 and Table 4). For flux measurements, model sensitivities have been pre-computed for every terrestrial grid cell and all PFTs that are available in the grid cell. When defining the site, the user can specify a mix among these PFTs. Uncertainties for data sampled at different sites and times are assumed to be uncorrelated. The uncertainty for each site is quantified by a standard deviation $\sigma(d)$, that reflects the combined effect of observational $\sigma\left(d_{\mathrm{obs}}\right)$ and model error $\sigma\left(d_{\bmod }\right)$ :

$\sigma(d)^{2}=\sigma\left(d_{\mathrm{obs}}\right)^{2}+\sigma\left(d_{\mathrm{mod}}\right)^{2}$

The unit of the data uncertainties depends on the data type. For flask and continuous samples of atmospheric $\mathrm{CO}_{2}$ it is $\mathrm{ppm}$, for eddy flux measurements it is $\mathrm{gC} \mathrm{m}^{-2} \mathrm{day}^{-1}$ (where $\mathrm{gC}$ stands for grams of carbon). The output of the network designer is the list of posterior uncertainties $\sigma(y)$ of the target quantities according to Eq. (2). $\sigma\left(y_{\bmod }\right)$ can be specified by the user as a percentage of the $20 \mathrm{yr}$ average of annual mean NPP.

\section{Experimental setup}

We will be evaluating several networks. To define these networks we have to select the sampling locations and the respective data uncertainties. Data uncertainty is generally difficult to estimate, especially in advance of actual measurements. In the following we give some motivation for our choices and for some cases we will test the effect of an alternative choice in Sect. 4.

The thrust of our study is the interaction between the spatial density of various classes of measurements and assumed heterogeneity of the spatial biosphere. It is important therefore that our choice of data uncertainty does not overly influence the results. We therefore make the most neutral possible choice of a uniform data uncertainty for each class of measurement. We also assume uncorrelated uncertainties in space and time. This is partly justified by the reduction in the underlying datasets to either daily or monthly means and, more importantly, by the focus of our study. We note that, in principle, systematic errors (biases) in the observations or the model (which would give rise to uncertainty correlations along the entire time series) can be removed or at least reduced by bias correction schemes. For example, Pillai et al. 


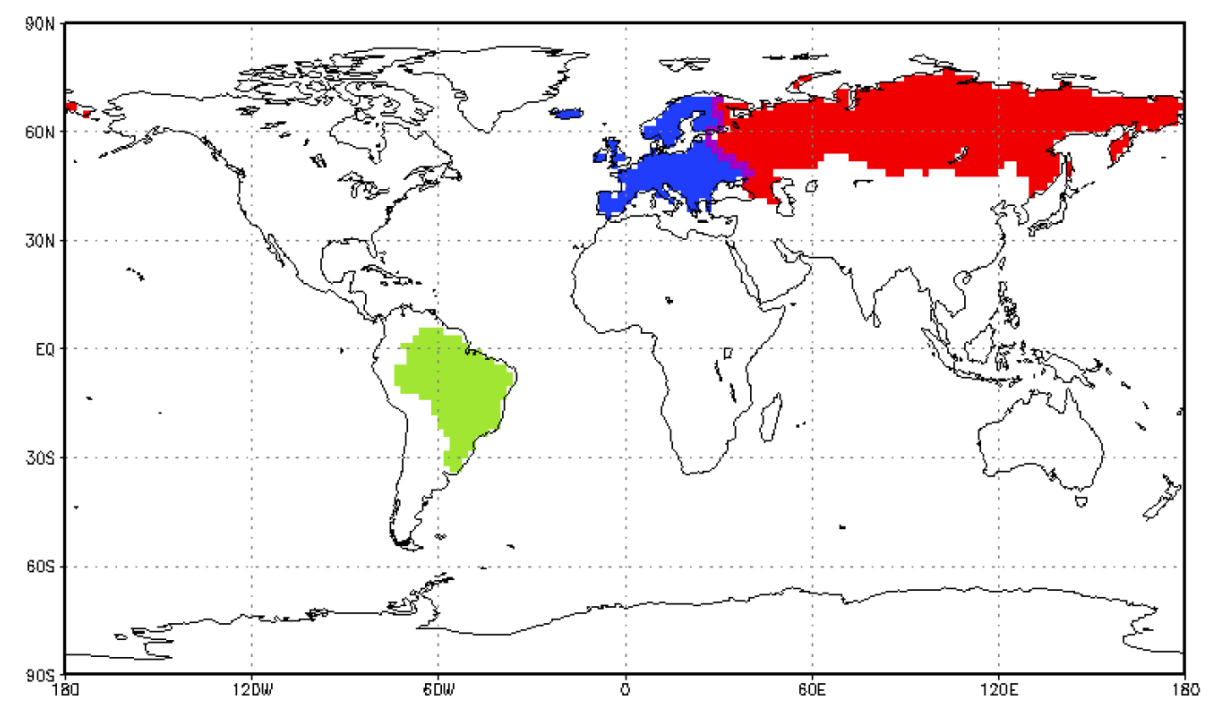

Fig. 2. Regions for computation of target quantities: grid cells contributing to fluxes over Europe (blue), Russia (red), both (violet), and Brazil (green).

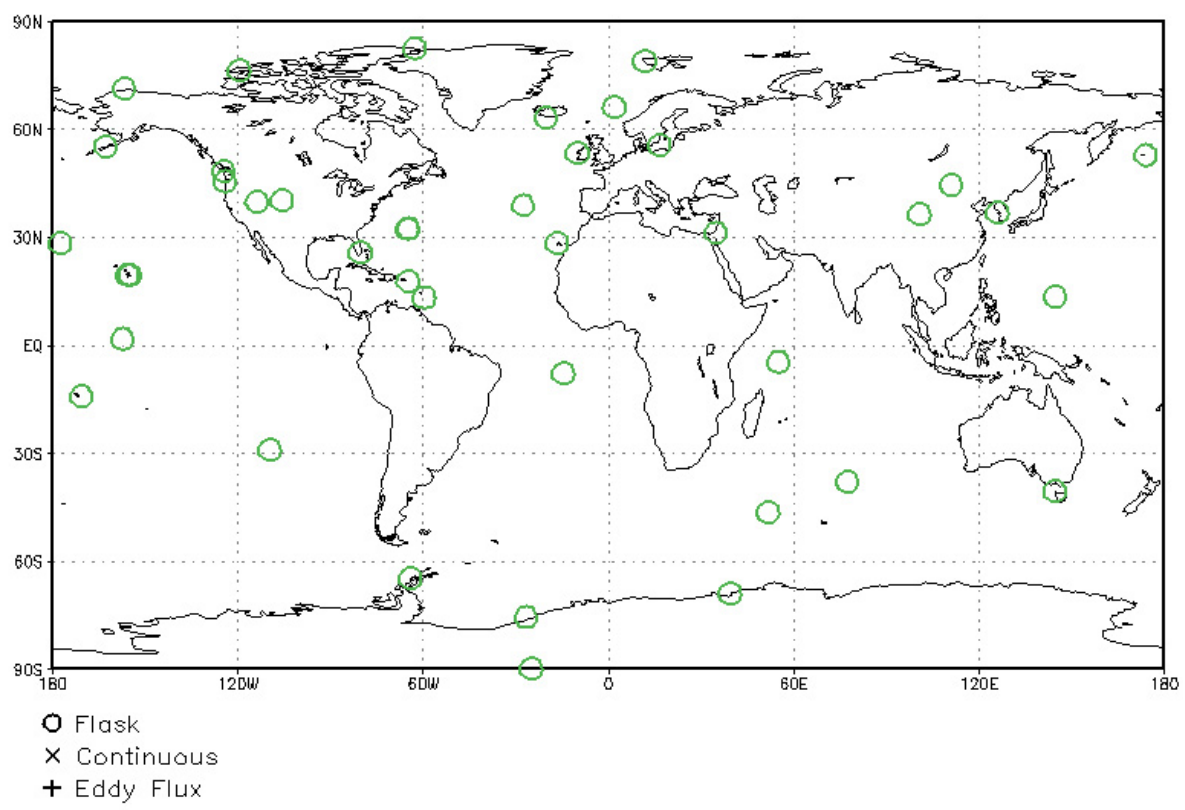

Fig. 3. Observational network flask providing atmospheric flask samples.

(2010) assess biases for the atmospheric data types and derive a recipe for their reduction.

For the flux measurements we use an uncertainty of $10 \mathrm{gCm}^{-2} \mathrm{day}^{-1}$. With respect to the minimum uncertainty of $3 \times 10^{-6} \mathrm{molm}^{-2} \mathrm{~s}^{-1} \approx 3.11 \mathrm{gCm}^{-2}$ day $^{-1}$ chosen by Knorr and Kattge (2005) this is a factor of about $\sqrt{10}$ larger. This effective sample size of 10 corresponds to ignoring half of the data because of nighttime sampling and allowing another factor of 5 to account of correlated uncertainties.

For the atmospheric data types we assume the combined error in the terrestrial and transport models to be the domi- nant contribution to data uncertainty. For flask samples (represented by monthly mean values) we use a data uncertainty of $1.0 \mathrm{ppm}$, above the average assigned by Rödenbeck et al. (2003) for the combined observational and transport model error. For continuous observations, which are more difficult to simulate, we use an uncertainty of $1.5 \mathrm{ppm}$. We can regard the factor of 1.5 compared to flask samples as an inflation of the data uncertainty, to achieve an effective sample size that is reduced by a factor of 2 . With roughly 30 times as many measurements this still gives continuous observations greater 


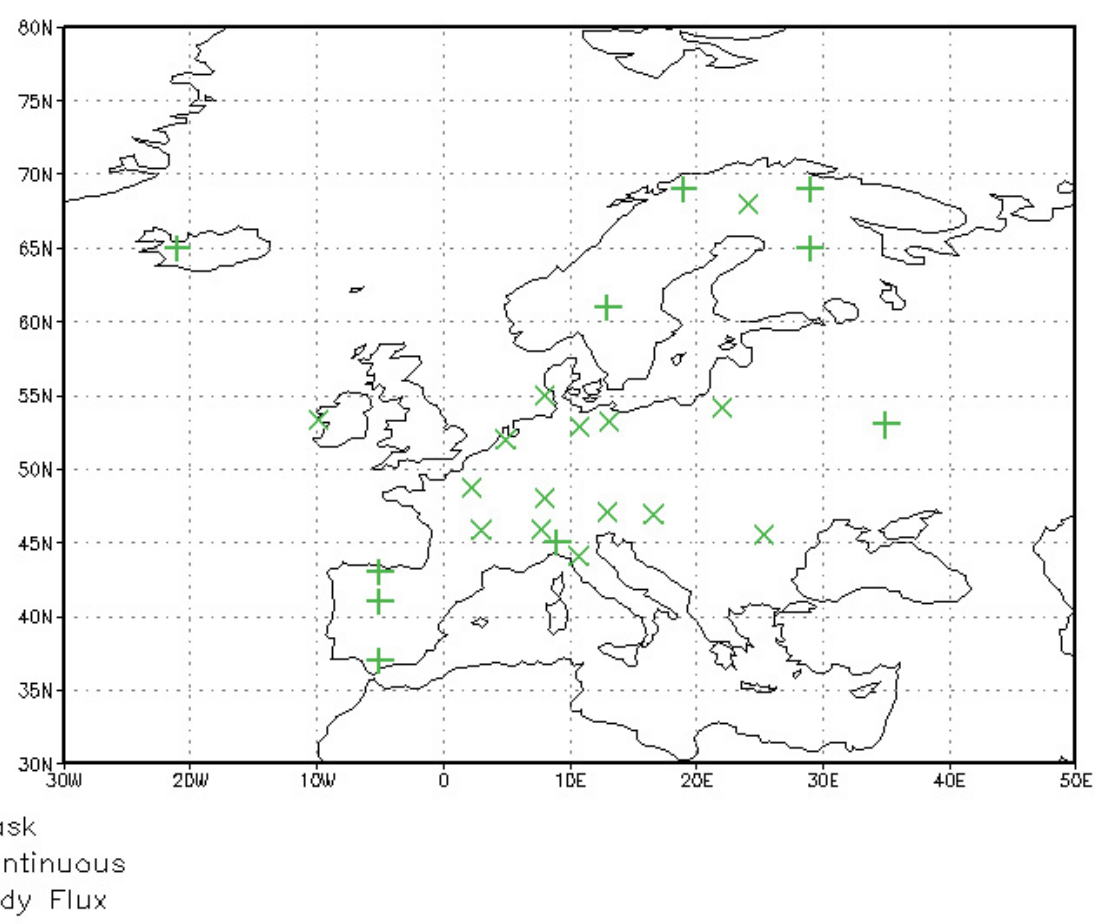

Fig. 4. Observational networks flux (+) providing flux measurements and cont $(\mathrm{X})$ providing continuous atmospheric samples.

weight than flask measurements but this is reasonable given their greater ability to represent a monthly mean.

Next we have to define the sampling locations. For each observational data type we define a base network:

- The atmospheric flask sampling network flask, which consists of the 41 monitoring stations listed in Table 2 of Kaminski et al. (2002) and shown in Fig. 3.

- The atmospheric continuous sampling network cont, which consists of the 15 sites listed in Table 4 and indicated with symbol "X" in Fig. 4.

- The eddy flux network flux, which consists of a dedicated site for each of the ten PFTs that are available to the model over Europe (PFT numbers 3-5 and 7-13 of Table 1). Each site is defined such that it is covered to $100 \%$ by the respective PFT. Table 3 lists the sites and Fig. 4 indicates their locations with the symbols "+".

We evaluate the networks in terms of the uncertainty reduction (Kaminski et al., 1999) in six target quantities:

$1-\frac{\sigma(y)}{\sigma\left(y_{\text {prior }}\right)}$,

where $\sigma\left(y_{\text {prior }}\right)$ denotes the uncertainty in the target quantity without any observational constraint and $\sigma(y)$ is taken from Eq. (2). The prior uncertainties for our target quantities are computed by propagating the prior parameter uncertainties of Scholze et al. (2007) via the Jacobian $N^{\prime}$ (Eq. 2). They are $0.45 \mathrm{GtC}, 1.45 \mathrm{GtC}$, and $1.13 \mathrm{GtC}$ for NEP over Europe, Russia, and Brazil, respectively, and $0.66 \mathrm{GtC}, 1.08 \mathrm{GtC}$, and $4.86 \mathrm{GtC}$ for NPP. $\sigma\left(y_{\bmod }\right)$ is an offset in Eq. (2). If the term was very high it would dominate the posterior uncertainty. To render the contrasts between the networks more drastic, we use a value of zero, i.e. we only analyse the effect of the networks on the parametric uncertainty in the target quantities. In fact, some of the parameters rather refer to the initial state, i.e. this source of uncertainty is also covered, to a limited extent, by our analysis.

In the above-described default set up BETHY runs with 13 PFTs. To investigate the robustness of the network performance with respect to model complexity in terms of the number of available PFTs, we extend the default set up as follows: we split the global vegetation into several equal fractions. Each fraction has its own set of 57 independent parameters with uncorrelated prior uncertainty. All fractions of a PFT share the location of the original PFT. In other words, a grid cell that in the default setup is populated by a single PFT is now composed of equal subgrid patches, each with their own PFT; the corresponding surface fluxes add up to one grid cell flux to be used for the atmospheric networks (hence the patches can be said to have the same location) but they are separately monitored by the flux network. In the following we will call the number of fractions multiplicity. With multiplicity 4 , for example, we have $4 \times 13=52$ PFTs and $57 \times 4=228$ parameters. A parameter that was global in the default configuration now has its validity restricted to one of the fractions of the global vegetation. A change 
Table 2. Process parameters, their symbols (2nd column), their description (3rd column), whether their scope is PFT specific or global (4th column), whether NEP (5th column) or NPP (6th column) are sensitive to them.

\begin{tabular}{llllrc}
\hline Number & Symbol & Description & scope & NEP & NPP \\
\hline 1 & $V_{\max }^{25}$ & maximum carboxylation rate $(\mathrm{C} 3)$ & $\mathrm{PFT}$ & $\mathrm{X}$ & $\mathrm{X}$ \\
2 & $a_{J, V}$ & ratio $V_{\max }^{25}$ over max electron transport $J_{\mathrm{max}}$ & $\mathrm{PFT}$ & $\mathrm{X}$ & $\mathrm{X}$ \\
3 & $\alpha_{q}$ & photon capture efficiency $(\mathrm{C} 3)$ & global & $\mathrm{X}$ & $\mathrm{X}$ \\
4 & $\alpha_{i}$ & quantum efficiency $(\mathrm{C} 4)$ & global & $\mathrm{X}$ & $\mathrm{X}$ \\
5 & $K_{\mathrm{C}}^{25}$ & Michaelis-Menten constant $\mathrm{CO}_{2}$ & global & $\mathrm{X}$ & $\mathrm{X}$ \\
6 & $K_{\mathrm{O}}^{25}$ & Michaelis-Menten constant $\mathrm{O}_{2}$ & global & $\mathrm{X}$ & $\mathrm{X}$ \\
7 & $a_{\Gamma, T}$ & temperature slope $\mathrm{CO}_{2}$ compensation point & global & $\mathrm{X}$ & $\mathrm{X}$ \\
8 & $E_{K_{\mathrm{O}}}$ & activation energy, $\mathrm{O}_{2}$ & global & $\mathrm{X}$ & $\mathrm{X}$ \\
9 & $E_{K_{\mathrm{C}}}$ & activation energy, CO & global & $\mathrm{X}$ & $\mathrm{X}$ \\
10 & $E_{V_{\max }}$ & activation energy, carboxylation rate $(\mathrm{C} 3)$ & global & $\mathrm{X}$ & $\mathrm{X}$ \\
11 & $E_{k}$ & activation energy, carboxylation rate $(\mathrm{C} 4)$ & global & $\mathrm{X}$ & $\mathrm{X}$ \\
12 & $E_{R_{\mathrm{d}}}$ & activation energy, dark respiration & global & $\mathrm{X}$ & $\mathrm{X}$ \\
13 & $f_{R, \text { leaf }}$ & leaf respiration ratio & global & $\mathrm{X}$ & $\mathrm{X}$ \\
14 & $f_{R, \text { growth }}$ & growth respiration ratio & global & $\mathrm{X}$ & $\mathrm{X}$ \\
15 & $f_{\mathrm{S}}$ & fraction of fast soil decomposition & global & $\mathrm{X}$ & \\
16 & $\kappa$ & soil moisture exponential for soil respiration & global & $\mathrm{X}$ & \\
17 & $Q_{10, \mathrm{f}}$ & soil respiration temperature factor, fast pool & global & $\mathrm{X}$ & \\
18 & $Q_{10, \mathrm{~s}}$ & soil respiration temperature factor, slow pool & global & $\mathrm{X}$ & \\
19 & $\tau_{\mathrm{f}}$ & fast pool soil carbon turnover time & global & $\mathrm{X}$ & \\
20 & $\beta$ & net CO ${ }_{2}$ sink factor & PFT & $\mathrm{X}$ & \\
21 & $c_{0}$ & atmospheric concentration offset & global & & \\
\hline
\end{tabular}

Table 3. Network flux. First number in site name indicates model grid cell and second number PFT.

\begin{tabular}{|c|c|c|c|c|}
\hline Name & Included & $\begin{array}{r}\text { Uncertainty } \\
{\left[\mathrm{gCm}^{-2} \text { day }^{-1}\right]}\end{array}$ & lon & lat \\
\hline site1413-3 & yes & 10.0 & 9.0 & 45.0 \\
\hline site1025-4 & yes & 10.0 & 35.0 & 53.0 \\
\hline site143-5 & yes & 10.0 & 19.0 & 69.0 \\
\hline site148-7 & yes & 10.0 & 29.0 & 69.0 \\
\hline site1495-8 & yes & 10.0 & -5.0 & 43.0 \\
\hline site1731-9 & yes & 10.0 & -5.0 & 37.0 \\
\hline site $1578-10$ & yes & 10.0 & -5.0 & 41.0 \\
\hline site377-11 & yes & 10.0 & -21.0 & 65.0 \\
\hline site388-12 & yes & 10.0 & 29.0 & 65.0 \\
\hline site621-13 & yes & 10.0 & 13.0 & 61.0 \\
\hline
\end{tabular}

of multiplicity also affects the prior uncertainty in the target quantities. Introducing the multiplicity $m$ means that $m$ copies have to share the same area. Hence, compared to the original flux $y$ the flux $y_{i}$ from each copy ( $i$ counting the copies) is reduced by a factor of $m$. And with it the original flux uncertainty $\sigma\left(y_{\text {prior }}\right)$ is also reduced by a factor of $m$ for each copy $\sigma\left(y_{i, \text { prior }}\right)$. Since there is no correlation of the prior uncertainty among the copies, the total flux uncertainty $\sigma\left(y_{\text {prior }, m}\right)$ is the square root of the sum of squares:
Table 4. Network cont of continuous atmospheric sampling sites over Europe.

\begin{tabular}{llrrr}
\hline Name & Included & $\begin{array}{r}\text { Uncertainty } \\
\text { [ppm] }\end{array}$ & lon & lat \\
\hline CBW & yes & 1.5 & 4.9 & 52.0 \\
CMN & yes & 1.5 & 10.7 & 44.1 \\
DGR & yes & 1.5 & 22.07 & 54.15 \\
FDA & yes & 1.5 & 25.3 & 45.47 \\
HUN & yes & 1.5 & 16.6 & 46.9 \\
MHD & yes & 1.5 & -9.9 & 53.33 \\
NGB & yes & 1.5 & 13.05 & 53.15 \\
PAL & yes & 1.5 & 24.12 & 67.97 \\
PRS & yes & 1.5 & 7.7 & 45.9 \\
PUY & yes & 1.5 & 3.0 & 45.8 \\
SAC & yes & 1.5 & 2.2 & 48.7 \\
SBK & yes & 1.5 & 12.98 & 47.05 \\
SCH & yes & 1.5 & 8.0 & 48.0 \\
WES & yes & 1.5 & 8.0 & 55.0 \\
WHF & yes & 1.5 & 10.77 & 52.8 \\
\hline
\end{tabular}

$$
\begin{aligned}
\sigma\left(y_{\text {prior }, m}\right) & =\sqrt{\sum_{i=1, m} \sigma\left(y_{i, \text { prior }}\right)^{2}}=\sqrt{\sum_{i=1, m}\left(\frac{\sigma\left(y_{\text {prior }}\right)}{m}\right)^{2}} \\
& =\frac{\sigma\left(y_{\text {prior }}\right)}{\sqrt{m}} .
\end{aligned}
$$




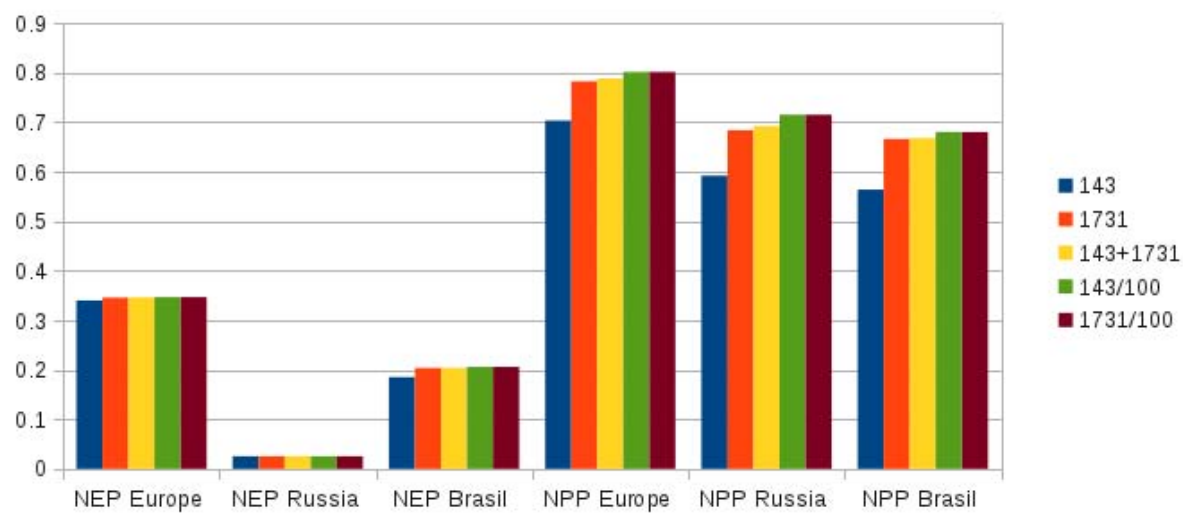

Fig. 5. Evaluation of two flux sites (blue and orange bars), of their combination (yellow bars), and of each site with data uncertainty reduced by a factor of 100 (green and brown bars): Uncertainty reduction for NEP and NPP integrated over three regions.

This means, for example, quadrupling the multiplicity halves the prior uncertainty.

\section{Results and discussion}

We start this section with evaluations of simple networks composed of one or two flux sites. Then we move on to the base networks defined in Sect. 3 and, finally, study the effect of increasing the number of PFTs that are available to the model.

\subsection{Simple configurations of flux sites}

The selection of a site location for sampling a particular PFT defines the Jacobian matrix that provides the link from the model parameters (required for simulating that PFT) to the simulated flux. To understand the effects which we will later see in larger networks, it is instructive to evaluate first a series of small networks consisting of one or two flux sites. We start with the separate evaluation of two sites which both observe PFT 9 (C3 grass) to $100 \%$, namely "site1731-9" in Southern Spain and "site143-9" in Northern Scandinavia. Note that we can populate any given location with up to three PFTs. For the current experiment we take the location of "site143-5" from Table 3 but populate it to $100 \%$ with PFT 9. For convenience, for the remainder of this subsection, we will refer to the sites just as "143" and "1731". The respective uncertainty reductions are displayed by blue (site "143") and orange (site "1731") bars in Fig. 5. First we note that flux measurements over Europe can reduce the uncertainty of target quantities over Russia and Brazil. This reflects our assumption of fundamental processes with a combination of universal and PFTspecific parameters: an observation provides information beyond its sampling time and location helping to reduce uncertainty everywhere. Figure 6 shows for site "1731" the uncertainty reduction in NEP per grid cell. This quantifies how the observational information of the site is spread around the globe. Comparing with Fig. 1 we note high uncertainty reduction where the dominant PFT is $\mathrm{C} 3$ grass.

Among the two sites in terms of NEP site " 1731 " performs only marginally better, but in terms of NPP it performs about 10 percentage points better ${ }^{1}$. Next we investigate the complementarity between the two sites, i.e. we use a network that consists of both sites and note a slight improvement for NPP over Europe and Russia (yellow bar in Fig. 5) compared to the better site "1731" alone. For these two target quantities the weaker site " 143 " is not redundant in this two site network, because it brings at least a little bit of extra information. In other words there is at least a slight complementarity between the two sites with respect to the two target quantities.

For the analysis of the above effects, recall that each scalar target quantity is (through the vector $\boldsymbol{N}^{\prime}$ of Eq. 2) influenced by its own one-dimensional sub-space of the parameter space, i.e. a target direction in parameter space. Likewise each scalar observation constrains a direction in parameter space (observed direction). We can use the analogy of a perspective under which the target direction is observed. If the target and observed directions are orthogonal, the observation can not reduce the uncertainty in the target quantity. If both directions are collinear, i.e. in the same subspace of the parameter space, the observation can most efficiently reduce the uncertainty in the target quantity. This means, for example, that even a hypothetically perfect measurement that removed all uncertainty for all parameters pertinent to one PFT would not completely constrain any of our target quantities (which are all influenced by several PFTs). In other words a one-site flux network is incomplete with respect to our target quantities. The strength of an observational constraint on a target quantity depends (1) on the sensitivity of the observed

\footnotetext{
${ }^{1}$ The unit percentage point quantifies an absolute change in the percentage value. For example, for a value of $40 \%$ an increase by 50 percentage points yields $90 \%$. By contrast, an increase by $50 \%$ corresponds to 20 percentage points and yields $60 \%$.
} 


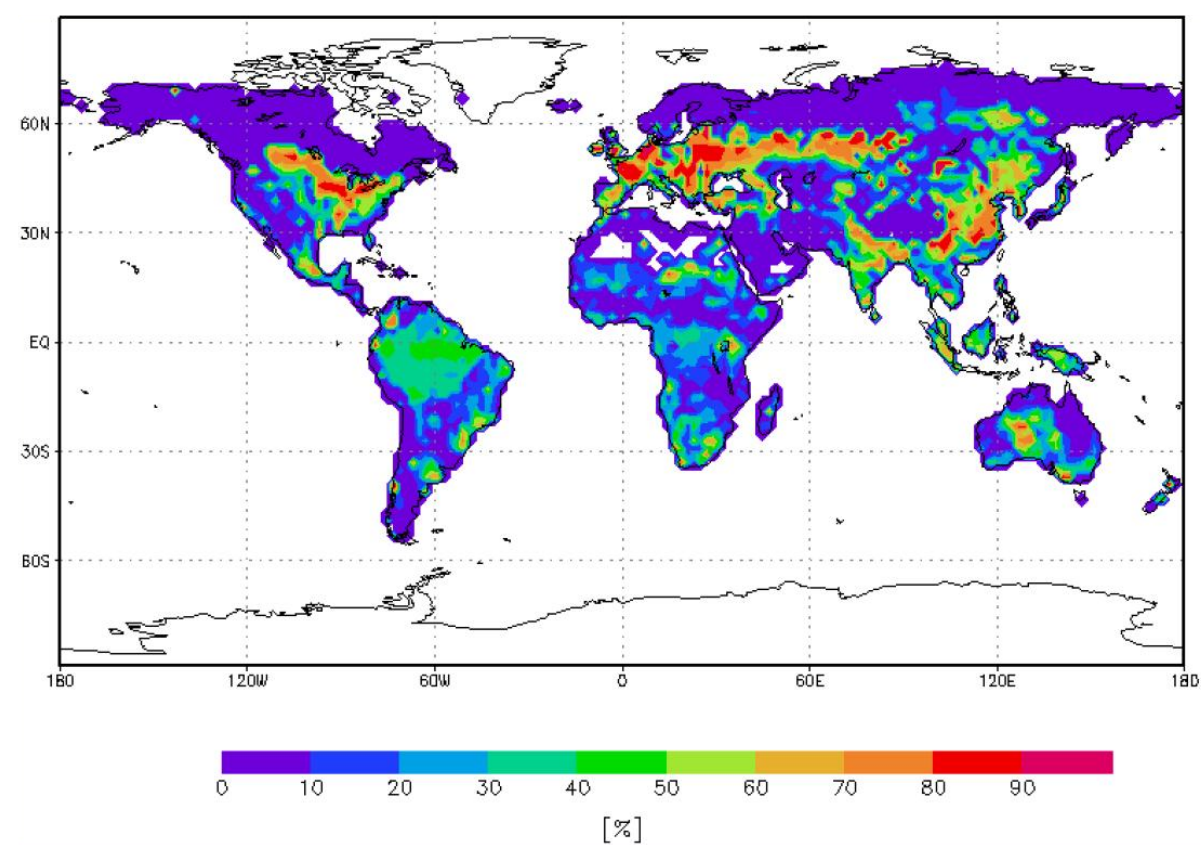

Fig. 6. Uncertainty reduction in NEP per grid cell, for a network consisting of a single flux site (site 1731-9 in Table 4).

quantity to a parameter change in the observed direction (signal size), (2) on how well the observed direction projects onto the target direction (perspective), and (3) on the data uncertainty. We use the same data uncertainty for both sites and the same target directions. The observed direction and signal size depend (1) on the PFT, (2) on the sampling time, and on (3) the meteorological driving data. Our two flux sites provide measurements at the same times (hourly for $20 \mathrm{yr}$ ) and of the same PFT. The only different factors are the meteorological driving data. Indeed the meteorology in Southern Spain is quite different from Scandinavia.

To isolate the effects of the perspective and the signal size on performance of the individual sites we reduce their respective data uncertainties by a factor of 100 (green and brown bars in Fig. 5). This can compensate for a weaker signal but does not change the perspective. Now both sites show exactly the same performance, i.e the Scandinavian site has just a smaller signal. In other words, we find the relevant information at both sites, but at sites with a larger signal we can afford a larger data uncertainty or, probably, a shorter observational period.

A common property between all networks evaluated in Fig. 5 is the larger uncertainty reduction for NPP compared to NEP. This happens although we sample hourly NEP, i.e. we should match the perspective for long-term NEP quite well. On the other hand, the target space for NPP has fewer dimensions, because it depends on fewer parameters. The extra parameters in NEP play an important role. This effect would probably be even more pronounced if NEP was compared with the Gross Primary Productivity (GPP) which is influenced by even fewer parameters (Koffi et al., 2012). Another point to note is that for Brazil the prior uncertainty in NPP is about four times higher than for NEP, and thus easier to reduce.

\subsection{Base networks and their combinations}

The performance of the three base networks flask (blue bars), cont (orange bars), and flux (yellow bars) is shown in Fig. 7. Over Europe, the flux network achieves an uncertainty reduction of about $99 \%$ for both NEP and NPP and outperforms both atmospheric networks. The reason for the strong performance of $f l u x$ over Europe is its completeness with respect to the European target quantities, i.e. the fact that for each PFT over Europe it contains a dedicated site. With respect to the Brazilian target quantities, in turn, the network flux is incomplete because it does not cover the tropical PFTs. This is why flux is weaker than the global network flask, in particular for NEP where the performance difference between both networks is over 50 percentage points.

The above suggests we would always attempt complete flux networks. In reality this will be hard to achieve, because we do not know how many PFTs are required to simulate the terrestrial carbon cycle, nor do we know their spatial distribution (Groenendijk et al., 2011). Hence, it may happen that we accidently miss a PFT in our flux network. We can test the effect of this by removing from network flux the site "17319" (network flux-C3). The performance over Europe drops by about 69 percentage points for NEP and 58 for NPP (green bars). Over Brazil the effect of missing the $\mathrm{C} 3$ grass site is only marginal (performance drop of less than four percentage points for NEP and less than two for NPP). 


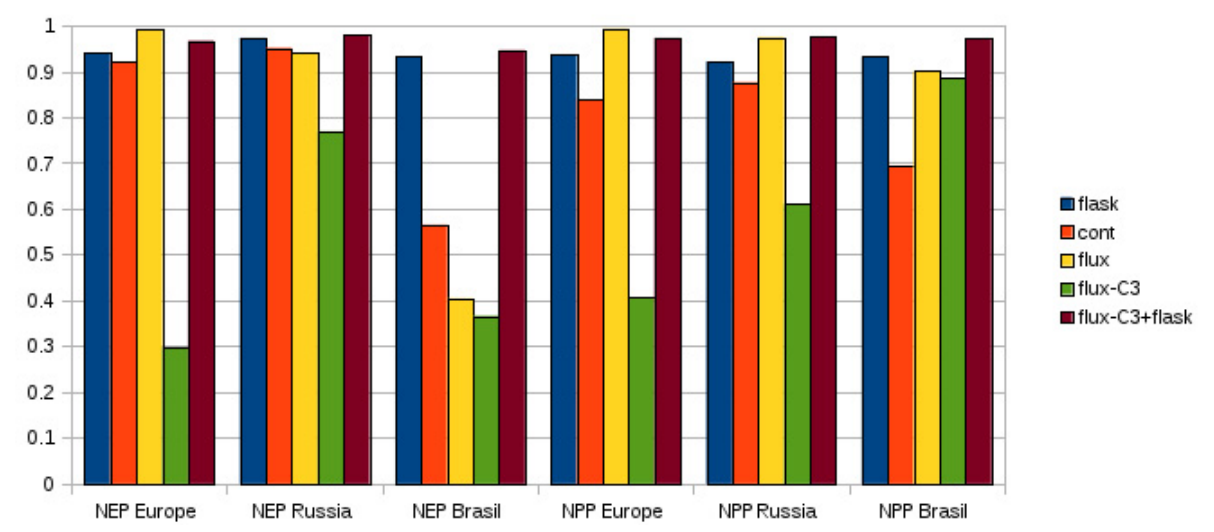

Fig. 7. Evaluation of three base networks, a flux network $f l u x-C 3$ that is incomplete over Europe and the combined $f l u x$ - $C 3+f l a s k$ network.

For the atmospheric networks, flask outperforms cont over Europe by 2 and 10 percentage points for NEP and NPP despite the European focus of cont. Obviously, for the atmosphere, the large-scale information matters. For Brazil or Russia it is not surprising that the global network flask is more powerful than the network cont. The most important aspect is that the atmospheric networks outperform the incomplete flux network flux-C3. The only exception is NPP over Brazil, where the loss of $\mathrm{C} 3$ had only a marginal effect on the performance of the flux network and $f l u x-C 3$ is stronger than cont but not than flask. We note that the relatively coarse resolution of TM2 may yield a slight overestimation in the integrative capacity of flask. For any given monthly mean sample, the higher resolution of LMDZ would resolve a finer influence structure (footprint) within the TM2 grid cells. On the other hand, our sampling period of $20 \mathrm{yr}$ would probably average out much of this time-dependent fine-scale structure, a mechanism that tends to increase the footprint. Over that period, it is not clear, per se, which of the transport models has a higher integrative capacity. We note, however, that in an inversion study the use of a high resolution model is favourable in order to minimise biases through resolutiondependent effects caused, e.g. by orography (see, e.g. Pillai et al., 2010). Increasing the data uncertainty of cont by a factor of four (to a value comparable to the data uncertainty of flask) yields only small performance reductions of 3 percentage points over Europe, 6-7 percentage points over Russia and below one percentage point over Brazil (not shown).

To assess the complementarity of atmospheric and flux networks, we combine the networks flux-C3 and flask. Over Europe the resulting network flux-C3 + flask performs almost as well as the complete flux network flux, and over Brazil and Russia even better. Both networks (flux-C3 and flask) complement each other. Given the experience from the two grass sites we evaluated initially (Sect. 4.1), we can think of the atmospheric network as an observer of averages over multiple sites. We can regard its addition to the flux network as an insurance against the incompleteness of the flux network.
What can we do in the case where we can not afford enough sites to sample all PFTs over our target region? Is it useful to have a flux site which observes two PFTs? We test this by removing the site "site1731-9" from the network flux and modify the PFT fractions at site "site143-9" to 50\% each for PFTs 5 and 9. This network has the same number of sites as $f l u x-C 3$ but much better performance (not shown). Uncertainty reduction for NEP over Europe is $76 \%$, and for the other target quantities the performance is only marginally (less than one percentage point) inferior to flux. This performance enhancement is based on the same principle as atmospheric sampling, the integration of a multi-PFT signal. This result seems surprising. It arises from the ability of a long time series to observe the different dynamics of the two underlying PFTs.

We can also investigate the complementarity of the base networks. Since the uncertainty reduction for the network flux is above $99 \%$ already, in this set of assessments we rather quantify the performance gain by the reduction in posterior uncertainty relative to the posterior uncertainty of $f u x$. Adding both atmospheric networks to network flux reduces NEP uncertainty over Europe by over $30 \%$ and NPP by $20 \%$. For the other regions the effect is much larger (up to $99 \%$ reduction for NEP over Brazil).

\subsection{Increased model complexity}

The above network evaluations are based on the default model setup with 13 PFTs. In the following we investigate the robustness of the results with respect to model complexity in terms of the number of available PFTs. To increase the number of PFTs we use the procedure described in Sect. 3.

For multiplicity 4, Fig. 8 shows the performance of the three base networks. Among the atmospheric networks flask is superior to cont for all target quantities, except for NEP over Europe where flask is slightly inferior. The network flux, in turn, is superior to flask except for NEP over Brazil and Russia. We define the network M4-1 flux, which is incomplete over Europe, by excluding one parameter copy out of 


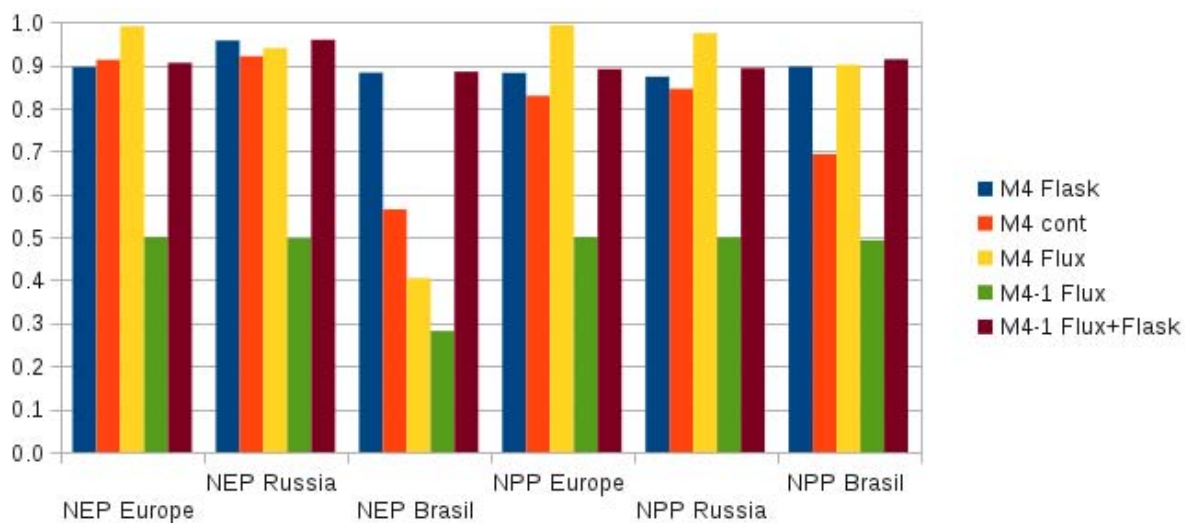

Fig. 8. Evaluation for multiplicity 4 of three base networks, an incomplete flux network with one copy of each PFT unsampled, and the combination of the incomplete flux network with the flask network.

the 4 from the network flux. This means M4-1 flux samples 30 out of the 40 PFTs that are available over Europe. As in the case of multiplicity 1 , the incompleteness is reflected in a strong drop in uncertainty reduction in particular over Europe, where the performance is roughly halved (green bars in Fig. 8). Combining M4-1 flux with flask is only marginally superior to flask alone. Apparently M4-1 flux is too incomplete to bring extra information. Put another way, the unobserved parts of the domain dominate the final uncertainty.

For multiplicity 25 (not shown), flux achieves uncertainty reductions close to $100 \%$ over Europe and above $85 \%$ elsewhere. We define two incomplete flux networks over Europe, one with one parameter copy out of 25 removed (over Europe 240 of 250 PFTs sampled) and the other one with two parameter copies out of 25 removed (230 PFTs sampled). Over Europe, compared to $f l u x$, the first network suffers a performance drop of about 20 percentage points, and the other one of almost 30 percentage points. Over Europe the flask performance (78\% for NEP and $74 \%$ for NPP) lies in-between both incomplete flux networks, which also holds for NPP over Russia. Elsewhere flask is better than the two networks. Even with a highly increased number of PFTs, an incomplete flux network that misses only a small fraction of the total PFTs is outperformed by flask. Combining flask with either one of the incomplete networks increases the flask performance over Europe by about ten percentage points for the smaller flux network and by another three percentage points for the larger network. This means both incomplete networks exhibit enough complementarity to flask to achieve a significant performance gain. Note that with multiplicity 25 the prior uncertainty is reduced by a factor of five (see Eq. 5). For example, an uncertainty reduction of $80 \%$ corresponds to the same posterior uncertainty as an uncertainty reduction of $96 \%$ (100-(100-80)/5\%) in BETHY's default setup (i.e. multiplicity 1). This means that the posterior uncertainty in NEP over Europe of flask (uncertainty reduction of $78 \%$ ) is similar to that in the default setup (uncertainty reduction of $94 \%)$.

\section{Conclusions}

QND is well-suited to explore the performance of observational networks of the carbon cycle. The network designer is a fast and easy-to-use QND implementation, that enables interactive network evaluations, e.g. within a meeting. Its current focus is on the continental-scale carbon balance. Figures 2, 3 and 5 in this paper are directly obtained from the network designer.

As mentioned above, the particular performance values are consequences of specific choices such as prior and data uncertainty, or the complexity of the underlying terrestrial model. There are, however, a set of general findings that follow from the above-mentioned assumption of fundamental equations that govern the processes controlling the terrestrial carbon fluxes. First, for direct flux observations, it is important to cover the full range of different PFTs and not the range of climates to which a given PFT is exposed. An incomplete flux network, i.e. one that misses a fraction of the PFTs risks a considerable performance loss. Atmospheric measurements are less prone to this problem, thus we can say that flux networks are more powerful while concentration networks are more robust. The combination can provide both qualities, i.e. atmospheric and flux networks complement each other.

The implications for the design of integrated observing strategies for the continental carbon balance seem clear. The baseline requirement is an atmospheric sampling network. That way if we underestimate the heterogeneity we will not find ourselves suddenly terribly undersampled. The strongest constraint, however, will come by overlaying this with a flux network which is as comprehensive as possible. Oversampling important PFTs will also give a diagnostic of heterogeneity. If parameters retrieved from one flux site enable us 
to predict the fluxes at a second then these are properly considered the same PFT for CCDAS, otherwise we need to increase the multiplicity.

The above assumption of fundamental equations that govern the processes controlling the terrestrial carbon fluxes does not apply to atmospheric transport inversions, which has an effect on the optimal sampling strategy. For example, transport inversions can incorporate the response of the carbon fluxes to a difference in climate only to a limited extent through their prior fluxes. Thus an optimal network for transport inversions needs to be capable of sampling the "climate space" if it wishes to capture this response.

This study addressed parametric and, to a certain extent, initial value uncertainty. To resolve structural uncertainty, it is important to build into the network the flexibility to detect features that are not or badly included in the model, i.e. the capability to discover surprises. Here, we have focused on carbon dioxide fluxes, however, observational networks for other trace gases, e.g. methane, can be evaluated with the same approach. Also, it is possible to evaluate networks that combine observations from space with in situ measurements as shown by Kaminski et al. (2010) and Kaminski et al. (2012). Similarly the column integrated $\mathrm{CO}_{2}$ measurements collected by the Total Carbon Column Observing Network (TCCON, http://www.tccon.caltech.edu/) can be included, as an extra data type, in the network designer. The approach can also be extended to oceanic networks.

Acknowledgements. We thank Han Dolman and Antoon Meesters as well as Christoph Gerbig for their reviewer comments and Dietrich Feist for his interactive comment. These comments improved the manuscript significantly. This work was supported in part by the European Community within the 6th Framework Programme for Research and Technological Development under contract no. 026188. PR is in receipt of an ARC Professorial Fellowship (DP1096309).

Edited by: M. Heimann

\section{References}

Carouge, C., Bousquet, P., Peylin, P., Rayner, P. J., and Ciais, P.: What can we learn from European continuous atmospheric $\mathrm{CO}_{2}$ measurements to quantify regional fluxes - Part 1: Potential of the 2001 network, Atmos. Chem. Phys., 10, 3107-3117, doi:10.5194/acp-10-3107-2010, 2010.

Carouge, C., Rayner, P. J., Peylin, P., Bousquet, P., Chevallier, F., and Ciais, P.: What can we learn from European continuous atmospheric $\mathrm{CO}_{2}$ measurements to quantify regional fluxes - Part 2: Sensitivity of flux accuracy to inverse setup, Atmos. Chem. Phys., 10, 3119-3129, doi:10.5194/acp-10-3119-2010, 2010 b.

Enting, I. G.: Inverse Problems in Atmospheric Constituent Transport, Cambridge University Press, Cambridge, 2002.

Giering, R. and Kaminski, T.: Recipes for adjoint code construction, ACM T. Math. Software, 24, 437-474, 1998.
Groenendijk, M., Dolman, A. J., van der Molen, M. K., Leuning, R., Arneth, A., Delpierre, N., Gash, J. H. C., Lindroth, A., Richardson, A. D., Verbeeck, H., and Wohlfahrt, G.: Assessing parameter variability in a photosynthesis model within and between plant functional types using global fluxnet eddy covariance data, Agr. Forest Meteorol., 151, 22-38, 2011.

Gurney, K. R., Law, R. M., Denning, A. S., Rayner, P. J., Baker, D., Bousquet, P., Bruhwiler, L., Chen, Y.-H., Ciais, P., Fan, S., Fung, I. Y., Gloor, M., Heimann, M., Higuchi, K., John, J., Maki, T., Maksyutov, S., Masarie, K., Peylin, P., Prather, M., Pak, B. C., Randerson, J., Sarmiento, J., Taguchi, S., Takahashi, T., and Yuen, C.-W.: Towards robust regional estimates of $\mathrm{CO}_{2}$ sources and sinks using atmospheric transport models, Nature, 415, 626-630, 2002.

Hardt, M. and Scherbaum, F.: The design of optimum networks for aftershock recordings, Geophys. J. Int., 117, 716-726, 1994.

Hauglustaine, D. A., Hourdin, F., Jourdain, L., Filiberti, M. A., Walters, S., Lamarque, J. F., and Holland, E. A.: Interactive chemistry in the Laboratoire de Météorologie Dynamique general circulation model: description and background tropospheric chemistry evaluation, J. Geophys. Res., 109, D04314, doi:10.1029/2003JD003957, 2004.

Heimann, M.: The Global Atmospheric Tracer Model TM2, Technical Report No. 10, Max-Planck-Institut für Meteorologie, Hamburg, Germany, 1995.

Kaminski, T. and Rayner, P. J.: Assimilation and network design, in: Observing the Continental Scale Greenhouse Gas Balance of Europe, edited by: Dolman, H., Freibauer, A., and Valentini, R., Ecological Studies, chap. 3, Springer-Verlag, New York, 2008.

Kaminski, T., Heimann, M., and Giering, R.: A coarse grid three dimensional global inverse model of the atmospheric transport, 1, Adjoint Model and Jacobian Matrix, J. Geophys. Res., 104, 18535-18553, 1999.

Kaminski, T., Knorr, W., Rayner, P. J., and Heimann, M.: Assimilating atmospheric data into a terrestrial biosphere model: a case study of the seasonal cycle, Global Biogeochem. Cy., 16, 1066, doi:10.1029/2001GB001463, 2002.

Kaminski, T., Scholze, M., and Houweling, S.: Quantifying the benefit of A-SCOPE data for reducing uncertainties in terrestrial carbon fluxes in CCDAS, Tellus B, 62, 784-796, doi:10.1111/j.1600-0889.2010.00483.x, 2010.

Kaminski, T., Knorr, W., Scholze, M., Gobron, N., Pinty, B., Giering, R., and Mathieu, P.-P.: Consistent assimilation of MERIS FAPAR and atmospheric $\mathrm{CO}_{2}$ into a terrestrial vegetation model and interactive mission benefit analysis, Biogeosciences, 9, 3173-3184, doi:10.5194/bg-9-3173-2012.

Knorr, W.: Annual and interannual $\mathrm{CO}_{2}$ exchanges of the terrestrial biosphere: process-based simulations and uncertainties, Global Ecol. Biogeogr., 9, 225-252, 2000.

Knorr, W. and Heimann, M.: Uncertainties in global terrestrial biosphere modeling: 1. A comprehensive sensitivity analysis with a new photosynthesis and energy balance scheme, Global Biogeochem. Cy., 15, 207-225, 2001.

Knorr, W. and Kattge, J.: Inversion of terrestrial biosphere model parameter values against eddy covariance measurements using Monte Carlo sampling, Glob. Change Biol., 11, 1333-1351, 2005.

Koffi, E., Rayner, P. J., Scholze, M., and Beer, C.: Atmospheric constraints on gross primary productivity and net flux: Results from 
a carbon-cycle data assimilation system, Glob. Biogeochem. Cy., 26, GB1024, doi:10.1029/2010GB003900, 2012.

Nijssen, B., Schnur, R., and Lettenmaier, D.: Retrospective estimation of soil moisture using the VIC land surface model, 19801993, J. Climate, 14, 1790-1808, 2001.

Pillai, D., Gerbig, C., Marshall, J., Ahmadov, R., Kretschmer, R., Koch, T., and Karstens, U.: High resolution modeling of $\mathrm{CO}_{2}$ over Europe: implications for representation errors of satellite retrievals, Atmos. Chem. Phys., 10, 83-94, doi:10.5194/acp-1083-2010, 2010.

Rayner, P. J., Scholze, M., Knorr, W., Kaminski, T., Giering, R., and Widmann, H.: Two decades of terrestrial Carbon fluxes from a Carbon Cycle Data Assimilation System (CCDAS), Global Biogeochem. Cy., 19, GB2026, doi:10.1029/2004GB002254, 2005.

Rayner, P. J.: The current state of carbon-cycle data assimilation, Current Opinion in Environmental Sustainability, 2, 289-296, doi:10.1016/j.cosust.2010.05.005, 2010.

Rayner, P. J. and O'Brien, D. M.: The utility of remotely sensed $\mathrm{CO}_{2}$ concentration data in surface source inversions, Geophys. Res. Let., 28, 175-178, 2001.

Rayner, P. J., Enting, I. G., and Trudinger, C. M.: Optimizing the $\mathrm{CO}_{2}$ observing network for constraining sources and sinks, Tellus B, 48, 433-444, 1996.

Rayner, P. J., Enting, I. G., Francey, R. J., and Langenfelds, R. L.: Reconstructing the recent carbon cycle from atmospheric $\mathrm{CO}_{2}$, $\delta^{13} \mathrm{C}$ and $\mathrm{O}_{2} / \mathrm{N}_{2}$ observations, Tellus B, 51, 213-232, 1999.
Rayner, P. J., Koffi, E., Scholze, M., Kaminski, T., and Dufresne, J.: Constraining predictions of the carbon cycle using data, Philos. T. Roy. Soc. A, 369, 1955-1966, doi:10.1098/rsta.2010.0378, 2011.

Rödenbeck, C., Houweling, S., Gloor, M., and Heimann, M.: $\mathrm{CO}_{2}$ flux history 1982-2001 inferred from atmospheric data using a global inversion of atmospheric transport, Atmos. Chem. Phys., 3, 1919-1964, doi:10.5194/acp-3-1919-2003, 2003.

Scholze, M., Kaminski, T., Rayner, P. J., Knorr, W., and Giering, R.: Propagating uncertainty through prognostic CCDAS simulations, J. Geophys. Res., 112, D17305, doi:10.1029/2007JD008642, 2007.

Solomon, S., Qin, D., Manning, M., Chen, Z., Marquis, M., Averyt, K., Tignor, M., and Miller, H.: IPCC, 2007: Climate Change 2007: The Physical Science Basis, Contribution of Working Group I to the Fourth Assessment Report of the Intergovernmental Panel on Climate Change, Cambridge University Press, Cambridge, United Kingdom and New York, NY, USA, 2007.

Wilson, M. F. and Henderson-Sellers, A.: A global archive of land cover and soils data for use in general-circulation climate models, J. Climatol., 5, 119-143, 1985. 